\title{
Pingyang Jiangya Fang pretreatment reduces the blood pressure of spontaneously hypertensive rats with Liver-Yang hyperactivity syndrome via ROS/Akt oxidative stress pathway
}

\author{
Zirong Li ${ }^{1 \#}$, Mengyao Wü ${ }^{2 \#}$, Deguo Liu ${ }^{1}$, Xiaoye Wang ${ }^{3}$, Wenli Yang ${ }^{4}$, Yuhong Wang ${ }^{1}$, Changjiang Xiao ${ }^{1,4}$ \\ ${ }^{1}$ State Key Laboratory of Chinese Medicine Powder and Medicine Innovation in Hunan (Incubation), Hunan University of Chinese Medicine, \\ Changsha, China; ${ }^{2}$ Zhuzhou Qianjin Pharmaceutical Co., Ltd., Zhuzhou, China; ${ }^{3}$ Department of General Surgery, Hunan Provincial Brain Hospital, \\ Changsha, China; ${ }^{4}$ Department of Cardiology, Affiliated Hospital of Hunan Academy of Traditional Chinese Medicine, Changsha, China \\ Contributions: (I) Conception and design: Z Li, Y Wang, C Xiao, W Yang; (II) Administrative support: Y Wang, C Xiao, X Wang; (III) Provision of \\ study materials: Y Wang, C Xiao; (IV) Collection and assembly of data: All authors; (V) Data analysis and interpretation: Z Li, D Liu, X Wang, M \\ Wu, W Yang; (VI) Manuscript writing: All authors; (VII) Final approval of manuscript: All authors. \\ \#These authors contributed equally to this work. \\ Correspondence to: Yuhong Wang. State Key Laboratory of Chinese Medicine Powder and Medicine Innovation in Hunan (Incubation), Hunan \\ University of Chinese Medicine, Changsha, China. Email: wyhyjy620@126.com; Changjiang Xiao. Department of Cardiology, Affiliated Hospital of \\ Hunan Academy of Traditional Chinese Medicine, Changsha, China. Email: changjiangx@163.com.
}

\begin{abstract}
Background: ROS/Akt pathway oxidative stress refers to a procedural response that activates various stress-sensitive signaling pathways in the cell thereby inducing insulin resistance (IR), and is closely related to high blood pressure. This study aims to investigate the effects of Pingyang Jiangya Fang (PYJYF) on the ROS/Akt pathway oxidative stress response in spontaneously hypertensive rats (SHRs) with Liver-Yang hyperactivity syndrome.
\end{abstract}

Methods: Except for the Wistar-Kyoto (WKY) and SHR groups, the other groups' rats were administered with Fuzi Decoction, to duplicate the model of hyperactivity of Liver-Yang. These rat's scleral color of deepens and become red, and rat's degree of irritability reached to above II degree. Compared with control group, rat's water consumption increased significantly, rotation tolerance time reduced significantly, pain threshold reduced significantly, rat tail vein systolic blood pressure (SBP) increased significantly. It shows that the model rats with hyperactivity of Liver-Yang have successfully replicated. Then, the corresponding drugs in the positive medicine group (candesartan cilexetil tablets) and the high, middle, and low dose groups of PYJYF (PYJYF-H, PYJYF-M, PYJYF-L) were administered. This work detected and analyzed behavior, SBP, renin-angiotensin system (RASS), expression of skeletal muscle angiotensin II type 1 receptor/angiotensin II type 2 receptor (AT1R/AT2R), IR index, renal/renal vascular/skeletal muscle tissue damage in each group. Moreover, we identified the target effect of point composition in the ROS/Akt signaling pathway.

Results: Unlike the WKY and SHR groups, the model group exhibited the phenotype of Liver-Yang hyperactivity syndrome, markedly increased SBP, hyper-RASS system, increased urine N-acetyl- $\beta$-Dglucosidase (NAG) and microalbuminuria (m-ALB), increased AT1R/AT2R ratio of skeletal muscle, IR, concomitant renal and skeletal muscle damage, as well as significant upregulation in the expression of ROS/ Akt signaling factor. PYJYF-H and PYJYF-M improved Liver-Yang hyperactivity syndrome, reduced SBP, the RASS system, urine NAG and m-ALB, AT1R/AT2R ratio, IR index, repair renal, renal vascular, and skeletal muscle tissue injury, as well as downregulated the expression of ROS/Akt signal factor.

Conclusions: PYJYF reduces blood pressure by inhibiting ROS/Akt pathway oxidative stress and reducing IR.

Keywords: Pingyang Jiangya Fang (PYJYF); ROS/Akt pathway; systolic pressure; insulin resistance (IR); oxidative stress 
Submitted Jul 06, 2020. Accepted for publication Nov 09, 2020.

doi: 10.21037/apm-20-1371

View this article at: http://dx.doi.org/10.21037/apm-20-1371

\section{Introduction}

Hypertension is currently one of the most prevalent cardiovascular diseases with an increasing incidence of nearly 1 billion people across the globe. Researchers have projected that due to the aging of population and sedentary lifestyle, the incidence of hypertension will surge to 1.5 billion people by 2025 (1). At present, hypertension remains a major risk factor for cardiovascular and cerebrovascular diseases, which can damage the structure and function of vital body organs including the heart, brain, and kidney. Without timely treatment, long-term illness causes serious and life-threatening complications such as stroke and myocardial infarction (2). As such, prevention and treatment of hypertension is critical.

Notably, there exist four types of antihypertensive drugs commonly used in clinical practice i.e., angiotensin receptor antagonists or invertase inhibitors, $\beta$-blockers, calcium channel blockers, and diuretics. The mechanism of essential hypertension on target organ damage is not yet available. When the antihypertensive effect fails to meet the Normal blood pressure range, it is often combined with multiple types of antihypertensive drugs until it affects glucose metabolism, damages $\beta$-cell function, and triggers diabetes before combining with hypoglycemic drugs. Also, increasing the financial burden on patients and their families triggers more physical and mental illness.

Pingyang Jiangya Fang (PYJYF) is a combination of Chinese classic prescription "Tianma Gouteng Yin Decoction" based on clinical experience. The prescription comprises traditional Chinese medicine, including Gypsophila, Uncaria, Dilong, etc. Of note, the reduce blood press clinical effect of PYJYF on hypertension and insulin resistance (IR) caused by Liver-Yang hyperactivity syndrome is significant (3). Previous research showed that this prescription exhibits acute and chronic antihypertensive effects on spontaneously hypertensive rat (SHR) models with Liver-Yang hyperactivity syndrome and it improved IR (4). Moreover, its antihypertensive effect might reduce prorenin (Pro), angiotensin II receptor (Ang II), aldosterone (Ald) levels, and inhibition of the renin-angiotensin system (RASS) activity (5). Nonetheless, specific signaling pathways of oxidative stress trigger IR and mediate hypertension and target organ damage.

Accumulating studies have revealed that IR is closely related to hypertension (3-6), increased blood pressure can increase IR, and long-term IR can increase blood sugar to form a vicious circle, so hypertension and IR interact with each other and promote each other (7). Additionally, recent studies (8) found that oxidative stress damage is a vital pathophysiological mechanism leading to hypertension and IR. It modifies various signal pathways and transcriptional regulation which influence glucose metabolism and damage $\beta$-cell function, eventually leading to IR. Occurrence and the generation of reactive oxygen species (ROS) is the fundamental incentive (9). For instance, ROS induces hypertension via the central and peripheral pathways, as well as participate in target organ damage (10).

In this experiment, we selected a model of SHR that closely resembles human essential hypertension (11), and copied into a rat model with the Liver-Yang hyperactivity syndrome model. Then, we observed their behavioral change, acute and chronic systolic blood pressure (SBP), RASS system index, IR index, pathological changes in renal, renal vessels, and skeletal muscle, as well as signal molecules related to the insulin conduction pathway, etc. This was geared towards observing the antihypertensive mechanism of SHR rat models with Liver-Yang hyperactivity syndrome.

We present the following article in accordance with the Animal Research Reporting of In Vivo Experiments (ARRIVE) reporting checklist (available at http://dx.doi. org/10.21037/apm-20-1371).

\section{Methods}

\section{Drugs and reagents}

Fuzi Decoction and PYJYF raw materials (concentrated into oral liquid $1.908 \mathrm{~g} / \mathrm{mL}$ ) were purchased from the Affiliated Hospital of Hunan Provincial Institute of Traditional Chinese Medicine. All the above mentioned medicinal materials were in a single batch. The composition of the PYJYF is shown in Table 1. Candesartan cilexetil was produced by Tianjin Takeda Pharmaceutical Co., Ltd. (batch number: J20150085, $8 \mathrm{mg} /$ tablet). The ROS detection kit was purchased from Nanjing Jiancheng Biotechnology Research Institute. Rabbit 
Table 1 Components of PYJYF formulation

\begin{tabular}{llc}
\hline Chinese name & Botanical name & Amount (g) \\
\hline Gui Zhen Cao & Bidentis Bipinnatae Herba & 15 \\
Gou Teng & Uncariae Ramulus Cum Uncis & 12 \\
Di Long & Pheretima & 10 \\
Shi Jue Ming & Concha Haliotidis & 20 \\
Zhen Zhu Mu & Concha Margaritifera & 12 \\
Ju Hua & Chrysanthemi Flos & 15 \\
Che Qian Zi & Plantaginis Semen & 10 \\
Gou Qi & Lycii Fructus & 12 \\
\hline
\end{tabular}

PYJYF, Pingyang Jiangya Fang (a prescriptions of traditional Chi nese medicine).

antirat phosphorylated serine/threonine kinase (p-Akt), serine/threonine kinase (Akt), glyceraldehyde-3-phosphate dehydrogenase (GAPDH) Doklon antibodies were purchased from Abcam (Abcam, UK). Trizol was purchased from American Amion company (Amion, USA). Reverse Transcription Kit was purchased from Thermo China official website (Thermo, USA). The reverse transcriptionpolymerase chain reaction (RT-PCR) kit was purchased from TaKaRa China official website (TaKaRa, Japan).

\section{Animals}

Nine to 10 weeks old SPF male SHR rats weighing $205.5 \pm 9.1$ g. Nine to 10 weeks old SPF male Wistar-Kyoto (WKY) rats weighing 204.4 \pm 7.2 g. Experimental animals were purchased from Beijing Vital River Laboratory Animal Technology Co., Ltd. (License number: 11400700148547, Beijing, China). All rats were placed in 5 animals/cages, and the temperature $\left(22 \pm 1{ }^{\circ} \mathrm{C}\right)$ and humidity $(55-65 \%$ relative humidity) were maintained for 1 week under a 14:10 light and dark cycle (light off at 15:00). All animal experiments were performed following the National Institutes of Health (NIH) Guidelines on the Use of Laboratory Animals and approved by the institutional committee on the care and use of animals of the First Affiliated Hospital of Hunan University of Traditional Chinese Medicine (Changsha, China). Notably, all efforts were expended to minimize animal suffering.

\section{Drug management}

Rats were acclimated for 5 days. WKY group rats were used as normal controls and fed normally. On the other hand, all SHR rats were randomly divided into six groups: SHR group, model group, positive medicine group, the high, middle, and low dose groups of PYJYF (PYJYF-H, PYJYF-M, PYJYF-L). SHR group was used as SHR control and fed normally. The other groups' rats were administered with Fuzi Decoction $20 \mathrm{~mL} / \mathrm{kg} / \mathrm{d}$ at 1 st week for 4 weeks, to duplicate the model of hyperactivity of Liver-Yang. After the model of hyperactivity of Liver-Yang was established. Positive medicine group rats administered by gavage candesartan cilexetil tablets suspension concentration $0.108 \mathrm{mg} / \mathrm{mL}$ at 5 th week for 8 weeks. PYJYF-H, PYJYF-M, PYJYF-L groups rats administered by PYJYF $1.908,0.954,0.477 \mathrm{~g} / \mathrm{mL}$ respectively at 5 th for 8 weeks. All experimental groups had only 10 rats.

\section{Behavioral test}

\section{General behavior observation}

First, we observed whether the coat color of the rat was shiny, the scleral color of both eyes deepened and was red, and changes in irritability. The degree of irritability was divided into three: (I) level I which refers to screaming and startling when holding the neck; (II) level II which refers to biting when holding the neck; and (III) level III which refers to screaming, startling, or even biting when lifting the tail, or when there is frequent fights among rats in the same cage. Notably, rats without the above conditions were considered as level 0 .

\section{Rotation time measurement}

The rotation tolerance time of rats was measured using a homemade rotation measuring instrument. Where the rats were placed on a self-made rotating platform. After stabilizing the rats, the rotary switch was turned on and the rotation speed set to $45 \mathrm{r} / \mathrm{min}$ then the time taken for the rats to fall was recorded. If the rotation time end, while the rat does not fall, then the experiment was stopped. This procedure was performed once a week.

\section{Measurement of water consumption in rats}

Before and after modeling, and when the drug intervention was about to end, for 3 consecutive days, $300 \mathrm{~mL}$ of drinking water was supplied per cage at 9 a.m every day, while the remaining water in each cage was measured at 9 a.m at next day. The value of total water intake in the cage was divided by the number of rats in each cage, this was the amount of water consumed by each rat on the day. Notably, 
the measurement was taken in 3 consecutive days, and the average value was taken as the amount of water consumed on that day.

\section{Determination of pain threshold}

A thermostatic water bath (Jintan Chengdong Xinrui instrument plant, China) was used to measure the pain threshold sensory temperature in rats at rest. The instrument was filled with water, and the temperature was kept above $40{ }^{\circ} \mathrm{C}$. Then, the instrument was placed in the beaker to preheat for 1 minute, and the rat was placed in the beaker. Subsequently, the temperature of the water bath was gradually raised and the temperature at which the rat was struggling (or screaming or jumping) was recorded within $10 \mathrm{~s}$. Then, the rats were taken out immediately. Of note, the temperature indicated by the water bath was the pain threshold of the rats. This measurement was recorded before and after modeling as well as after drug intervention.

\section{Arterial blood pressure measurement of rat's tail}

981-B electronic thermostat electric heating mantle (Tianjin Taisite Instrument factory), and RBP-1B rat tail artery manometer (Nanjing Debao Biochemical factory) noninvasive tail artery manometry method were used to measure the rat's tail artery SBP before and after modeling. On the first day of drug intervention, a sequential method was used to measure the $24 \mathrm{~h}$ acute hypotensive test at every $2 \mathrm{~h}$. After drug intervention, the SBP of each rat was measured every weekend. The rats were subjected to training three times before formal pressure measurement. SBP of each rat was measured three times and the average was taken. Precautions for pressure measurement included; before the measurement, the animal should be lifted to the laboratory for 30 minutes; avoid noise during the measurement; and keep the room temperature at $24-26^{\circ} \mathrm{C}$. Again, where applicable, grasp the rat and move gently to avoid irritating the rat. Notably, the sensor should be placed at the root of the rat's tail. After fixing the animal, the stool should be cleared immediately due to the stress reaction. Additionally, the measurement time should not be too long. The rat should be removed from the mouse bag if it is not detected for more than 30 minutes, then, let it stand for a while before measuring.

\section{Collection and processing of blood and tissue samples}

Blood was collected from the caudal vein venous blood collection before and after modeling, and was allowed to stand for $30 \mathrm{~min}$, then centrifuged at 3,000 r/min for $15 \mathrm{~min}$. The supernatant was obtained and stored in a $-80{ }^{\circ} \mathrm{C}$ refrigerator for later use. After the end of the dosing period, all rats were weighed after fasting for 12 hours without water. After the behavioral test, the rats were anesthetized intraperitoneally with $2 \%$ pentobarbital sodium $(3 \mathrm{~mL} / \mathrm{kg})$. The limbs of the rat were tentatively clamped with forceps where the rat showed no apparent response. Then, the abdominal cavity was cut open to expose the abdominal aorta. Blood was collected from the abdominal aorta using a blood preparation needle prepared in advance and placed in a centrifuge tube. After the blood sample was left for 30 minutes, it was centrifuged in a high-speed desktop centrifuge at 3,000 r/min for 15 minutes. Then, the centrifuge tube was carefully removed and the supernatant transferred to the labeled EP tube and stored in an $80^{\circ} \mathrm{C}$ refrigerator. After 1:40 dilution, based on the manufacturer's instructions, the plasma Pro, Ang II, Ald, fasting plasma glucose (FPG), fast insulin (FINs), resistin (RES) of each group of rats were passed through an enzyme-linked immunosorbent assay (ELISA) (Enzo Life Sciences, Plymouth, PA, USA).

The renals were removed on both sides. The lower limbs were severed, and the skin was quickly cut open. The quadriceps muscles were separated on an ice tray. The muscle surface fat was removed, and the blood was washed in iced physiological saline. Then, the water was dried with filter paper and weighed. The amount of phosphate buffer saline (PBS) solution was frozen in liquid nitrogen for subsequent experiments.

Histopathological observation of renal, renal vessels, and skeletal muscle: They were fixed with $10 \%$ formalin, then embedded in paraffin, sectioned $(5 \mu \mathrm{m})$, conventional Hematoxylin-eosin (HE) staining, and morphological changes observed under a light microscope.

\section{IR-related indicators}

The homa insulin-resistance (HOMA-IR) and RES have been considered as typical indicators of IR. ELISA was used to detect plasma FPG, FINs, and RES. HOMAIR was calculated based on HOMA-IR $=($ FPG $\times$ FINs $) /$ $22.5(11,12)$.

\section{RASS system-related indicators}

The RASS system is the renin-angiotensin-aldosterone system, which is an important endocrine system for regulating blood pressure in the human body. Pro, Ang II, 
Ald can be used to judge the prognosis of hypertension. ELISA was used to detect Pro, Ang II, and Ald. Notably, the kit instructions were referred for specific operations.

\section{Determination of urine $\mathrm{N}$-acetyl- $\beta$-D-glucosidase (NAG) and microalbuminuria (m-ALB)}

Reports indicate that urine NAG and $\mathrm{m}$-ALB are sensitive indicators of hypertension renal damage. Before the end of the drug intervention, rats were placed in a metabolic cage for free diet, then, 24 hours of urine was collected, it was centrifuged in a high-speed desktop centrifuge at $10,000 \mathrm{r} / \mathrm{min}$ for 15 minutes, and stored in a refrigerator at $-20{ }^{\circ} \mathrm{C}$ for testing. The urine NAG was measured via the ELISA, while the m-ALB was measured using the radioimmunoassay kit.

\section{Angiotensin II type 1 receptor/angiotensin II type 2 receptor (AT1R/AT2R) immunocytochemical staining of skeletal muscle}

Ang II is a critical part of the RASS system as well as the primary ligand of AT1R and AT2R, it exhibits a close affinity with the two. Blocking AT1R blocks the effect of angiotensin II, which in turn blocks the activity of the RASS system (13). Immunocytochemical staining was used to detect skeletal muscle AT1R/AT2R optical density (OD) values, and Image-Pro Plus 6.0 software was used to analyze the immunohistochemical method, where 6 slices of 200 times field of view were randomly selected for photographing in each section of each group. When taking photos, the entire field of vision should be filled with the organization, and the consistency in the background light of each photo should be maintained. Image-Pro Plus 6.0 software was used to select similar brown-yellow color as the uniform standard for judging the positives of all photos. Each photo was analyzed to obtain the cumulative integral optical density (IOD) value. The AT1R and AT2R IOD values of each group were recorded.

\section{Histology}

The renals, renal vessels, and skeletal muscles tissues fixed in $4 \%$ paraformaldehyde solution were embedded in paraffin and sliced in to small sections. The pieces were dewaxed, hydrated and thereafter stained with hematoxylineosin (H\&E) staining. The stained sections were then dehydrated, use xylene to make tissue transparent, and mounted on slides, then observed under the microscope at 200x magnification. Bioquant System Imaging Software
(Bioquant Image Analysis, Nashville, TN, USA) was used to detect the histopathological changes.

\section{Expression of ROS/Akt signal molecules in skeletal muscle}

Of note, oxidative stress activates the ROS/Akt signaling pathway, further damage the insulin molecular signaling pathway, and increase IR (14). The ROS content of skeletal muscle was measured using ELISA based on the kit instructions. Reverse transcription-polymerase chain reaction (RT-PCR) was used to detect the expression of Akt mRNA. The spare rat skeletal muscle was taken and the total RNA was extracted by Trizol method. Nucleic acid-protein concentration tester was used to measure the concentration and purity of RNA, then cDNA was amplified by reverse transcription i.e., $20 \mu \mathrm{L}$ of amplification system: $1 \mu \mathrm{L}$ of cDNA template, $1 \mu \mathrm{L}$ of upstream and downstream primers, $10 \mu \mathrm{L}$ of SYB solution, $7 \mu \mathrm{L}$ of enzyme-free water. The thermal cycling parameters included, $95^{\circ} \mathrm{C}$ predenaturation for $3 \mathrm{~min}$, amplification curve: $95^{\circ} \mathrm{C}, 10 \mathrm{~s} ; 58^{\circ} \mathrm{C}, 30 \mathrm{~s} ; 72^{\circ} \mathrm{C}, 10 \mathrm{~s}, 40$ cycles; melting curve: $95^{\circ} \mathrm{C}, 15 \mathrm{~s} ; 60{ }^{\circ} \mathrm{C}, 15 \mathrm{~s} ; 95^{\circ} \mathrm{C}, 15 \mathrm{~s}$. The Cq value of the PCR product of the target gene and $\beta$-actin was measured. The relative expression of the test gene was calculated using the $\mathrm{Cq}^{-2 \Delta \Delta}$ method. Use Primer Premier 6.0 Software designed the primers by Shanghai Biotech Bioengineering Co., Ltd. The primers included $\beta$-actin (110 bp): F, 5'-AGGGCTGCCTTCTCTTGTGA-3'; R, 5'-AACTTGCCGTGGGTAGAGTCA-3'. Akt (107 bp): F, 5'-TTGCCCACACGCTTACTGAG-3'; R, 5'-CATGACAAAGCAGAGGCGGT.

Western Blot was used to detect the expression of skeletal muscle p-Akt and Akt protein, while radioimmunoprecipitation (RIPA) and benzenesulfonyl fluoride protease inhibitor (Solarbio Technology, Beijing, PRC) were used to obtain the protein. Protein was obtained using RIPA and benzenesulfonyl fluoride protease inhibitor, then the protein concentration was analyzed using a nucleic acid-protein detector (BioDrop, UK). The protein (50 $\mu \mathrm{g})$ was electrophoresed on a sodium dodecyl sulfate (SDS) polyacrylamide gels at a constant voltage of $120 \mathrm{~V}$ for 100 minutes, then transferred to a polyvinylidene fluoride (PVDF) membrane (Millipore, MA). The membranes were incubated using different antibodies (1:500 monoclonal rabbit anti-p-Akt, Akt) and incubated at $4{ }^{\circ} \mathrm{C}$ overnight. Then, the membranes were incubated with appropriate secondary antibodies (1:5,000; Beijing Biosynthetic Biology Technology, Beijing, China) for 1 hour at room temperature 
on the next day. The immune response was quantified by scanning density meter (Quantity One software; BioRad), and the density of each band was normalized to its $\beta$-actin density.

\section{Statistical analysis}

Data were presented as means \pm SD. One-way analysis of variance (ANOVA) and $t$-tests were used for comparisons among and between groups, respectively. All analyses were conducted using SPSS 21.0. The difference at $\mathrm{P}<0.05$ was considered statistically significant.

\section{Results}

\section{Behavioral testing}

Notably, PYJYF exhibits a significant antihypertensive effect and improves the Liver-Yang hyperactivity syndrome. The degree of sclera redness and irritability depicted irritability and aggressive behavior. Figure $1 A, B$ shows the changes in the number of scleral red staining and irritability $\geq$ grade II: after modeling, except for the WKY and SHR groups, the number of scleral red staining in other groups increased significantly; while after drug intervention, the number of scleral red staining and irritability $\geq$ II level in the PYJYF-H, PYJYF-M and PYJYF-L decreased significantly. This was directly proportional to the dose of PYJYF, suggesting that PYJYF could alleviate the anxiety symptoms of LiverYang hyperactivity syndrome. Meanwhile, the amount of drinking water reflects the Yin and Yang imbalance caused by hyperactivity of the body and the lack of Yin fluid. Figure $1 C$ shows the changes in drinking water: after modeling, unlike the WKY and SHR groups, the drinking water levels of the other groups increased significantly $(\mathrm{P}<0.05$, $\mathrm{P}<0.01$ ); whereas after drug intervention, unlike the model group, the water consumption in the PYJYF-H, PYJYF-M and PYJYF-L was significantly reduced $(\mathrm{P}<0.05, \mathrm{P}<0.01)$, suggesting that the PYJYF improved the consumption of Yin fluid. The rotation time reflects the ability of the body to resist high blood pressure vertigo. Figure $1 D$ illustrates the change in rotation time: after modeling, in contrast with the WKY and SHR groups, the rotation time of the other groups was significantly reduced $(\mathrm{P}<0.01)$; while after drug intervention, unlike the model group, the rotation time of the PYJYF-H, PYJYF-M, PYJYF-L, and positive medicine group increased significantly $\mathrm{P}<0.05, \mathrm{P}<0.01$ ), implying that the PYJYF could improve dizziness caused by hypertension. The low pain threshold implies an excessively defensive self-protection response of the body. Figure $1 E$ demonstrates the changes in the pain threshold sensory temperature: after modeling, unlike the WKY and SHR groups, the pain threshold sensory temperature in the other groups was significantly reduced $(\mathrm{P}<0.05, \mathrm{P}<0.01)$; whereas after drug intervention, in contrast with the model group, the pain threshold sensory temperature of the PYJYF-H, PYJYF-M, PYJYF-L, and positive medicine group increased significantly $(\mathrm{P}<0.05, \mathrm{P}<0.01)$, indicating that the PYJYF enhanced the self-defense capability of the body. Figure $1 F$ reveals the changes in SBP: after modeling, compared with the WKY and SHR groups, the SBP in the other groups increased significantly $(\mathrm{P}<0.01)$; whereas after drug intervention, in contrast with the model group, SBP of the PYJYF-H, PYJYF-M, PYJYF-L, and positive medicine group was significantly reduced $(\mathrm{P}<0.01)$, meaning that PYJYF had a significant reduction effect on blood pressure.

\section{PYFYF inbibits IR}

Plasma FPG, FINs, and RES levels are directly related to IR and reflect the glucose metabolism of islet $\beta$ cells. Before treatment, compared with WKY and SHR groups, FPG, FINs, RES, and HOMA-IR in the other groups were significantly increased $(\mathrm{P}<0.01)$; while after treatment, unlike the model group, the FPG, FINs, RES, and HOMAIR in the PYJYF-H, PYJYF-M as well as the positive medicine groups were significantly decreased $(\mathrm{P}<0.01)$. Thus, these findings indicate that the expression of plasma FPG, FINs, RES, and HOMA-IR can be reversed by PYJYF (Figure $2 A, B, C, D$ ).

\section{Twenty-four bours acute bypotension test}

The SBP at every 2 hours after the first day of administration showed that the blood pressure of the model group showed an "antiscoop" rise. The positive medicine group and PYJYF-H, PYJYF-M, PYJYF-L significantly reduced the $\mathrm{SBP}$ to different degrees (Figure 2E).

\section{Eight-week chronic antihypertensive experiment}

The SBP of each group every weekend showed that the blood pressure in the model group showed a continuous increase. PYJYF-H, PYJYF-M, PYJYF-L reduced SBP to varying degrees, and PYJYF-H significantly reduced the blood pressure (Figure $2 F$ ). The antihypertensive effect was 

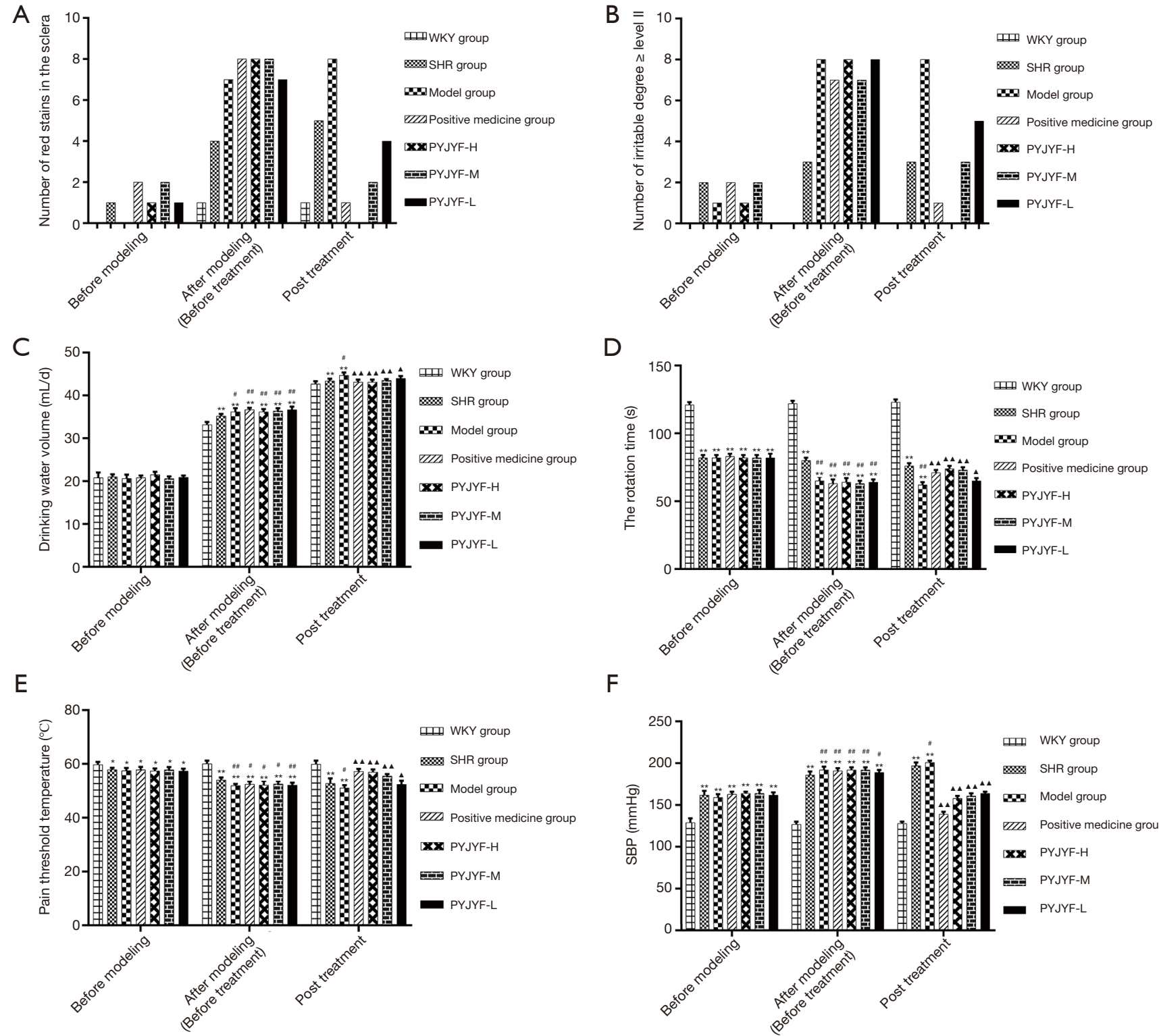

$\mathrm{F}$

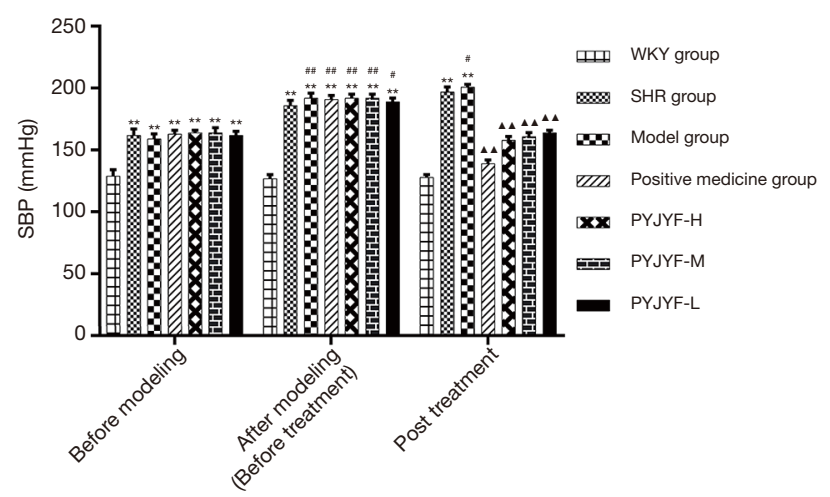

Figure 1 The behavioral test of rats in each group before modeling, after modeling (before treatment) and after treatment. (A) The number of sclera red staining in each group; (B) the number of rats with irritability II or higher degree in each group number; (C) the average daily water intake of each group of rats $(\mathrm{mL})$; $(\mathrm{D})$ the length of rotation time of each group of rats; (E) the pain threshold temperature of each group of rats $\left({ }^{\circ} \mathrm{C}\right)$; (F) SBP of rats in each group $(\mathrm{mmHg})$. All values are expressed as the means \pm SDs. Compared with WKY group, *, $\mathrm{P}<0.05,{ }^{* *}, \mathrm{P}<0.01$; compared with SHR group, ${ }^{\#}, \mathrm{P}<0.05,{ }^{\# \#}, \mathrm{P}<0.01$; compared with the model group, ${ }^{\Delta}, \mathrm{P}<0.05,{ }^{\Delta}{ }^{\Delta}, \mathrm{P}<0.01$. SBP, systolic blood pressure; WKY, Wistar-Kyoto rat (as a normal control); SHR, spontaneously hypertensive rats; PYJYF, Pingyang Jiangya Fang (a prescriptions of traditional Chinese medicine); PYJYF-H, PYJYF high dose group; PYJYF-M, PYJYF medium dose group; PYJYF-L, PYJYF low dose group. 

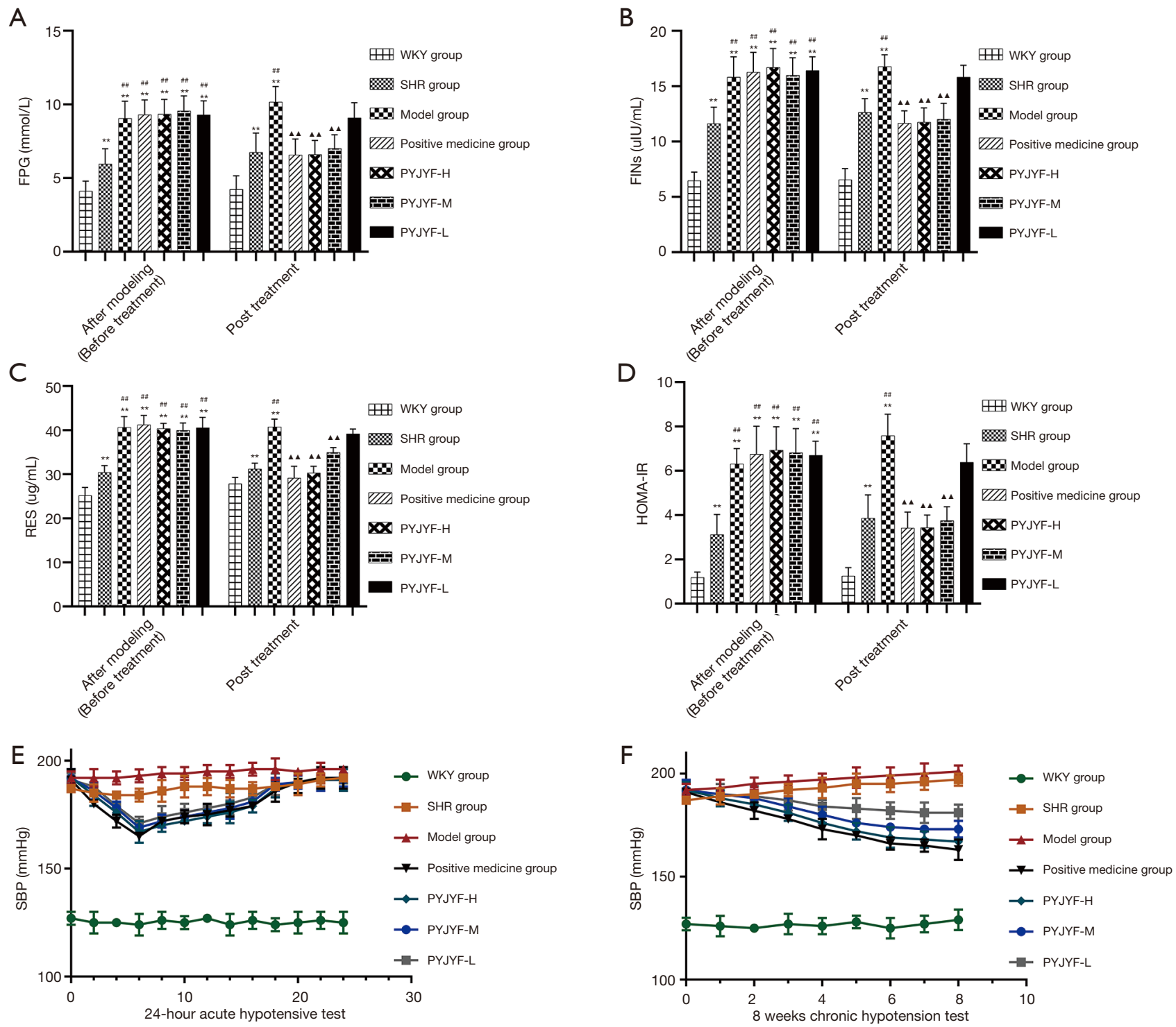

Figure 2 Effects of PYJYF on FPG, FINs, RES, HOMA-IR, 24 h acute blood pressure test and $8 \mathrm{w}$ chronic blood pressure test in rats. (A) FPG (mmol/L) before and after treatment in each group rats; (B) FINs (uIU/mL) before and after treatment in each group of rats; (C) RES (ug/mL) before and after treatment in each group of rats; (D) HOMA-IR before and after treatment in each group of rats; (E) 24 h rat SBP fluctuations $(\mathrm{mmHg})$; $(\mathrm{F}) 8 \mathrm{w}$ rat SBP changes $(\mathrm{mmHg})$. All values are expressed as the means \pm SDs. Compared with $\mathrm{WKY}$ group, * $\mathrm{P}<0.05$, **, $\mathrm{P}<0.01$; compared with SHR group, ${ }^{\#}, \mathrm{P}<0.05,{ }^{\#}, \mathrm{P}<0.01$; compared with the model group, $\stackrel{\boldsymbol{\Delta}}{ }, \mathrm{P}<0.05, \stackrel{\Delta}{\Delta}, \mathrm{P}<0.01$. FPG, fasting plasma glucose; FINs, fast insulin; RES, resistin; HOMA-IR, homa insulin-resistance; SBP, systolic blood pressure; WKY, Wistar-Kyoto rat (as a normal control); SHR, spontaneously hypertensive rats; PYJYF, Pingyang Jiangya Fang (a prescriptions of traditional Chinese medicine); PYJYF-H, PYJYF high dose group; PYJYF-M, PYJYF medium dose group; PYJYF-L, PYJYF low dose group.

similar to the positive medicine group.

\section{PYFYF reduces the AT1R/AT2R ratio}

Figure $3 A$ shows the expression of AT1R and AT2R in skeletal muscle via immunostaining. Figure $3 B$ shows that AT1R was significantly reduced in the PYJYF-H, PYJYF-M, and positive medicine groups $(\mathrm{P}<0.01)$. Figure $3 C$ demonstrates that AT2R increased significantly in the PYJYF-H, PYJYF-M, and positive medicine 

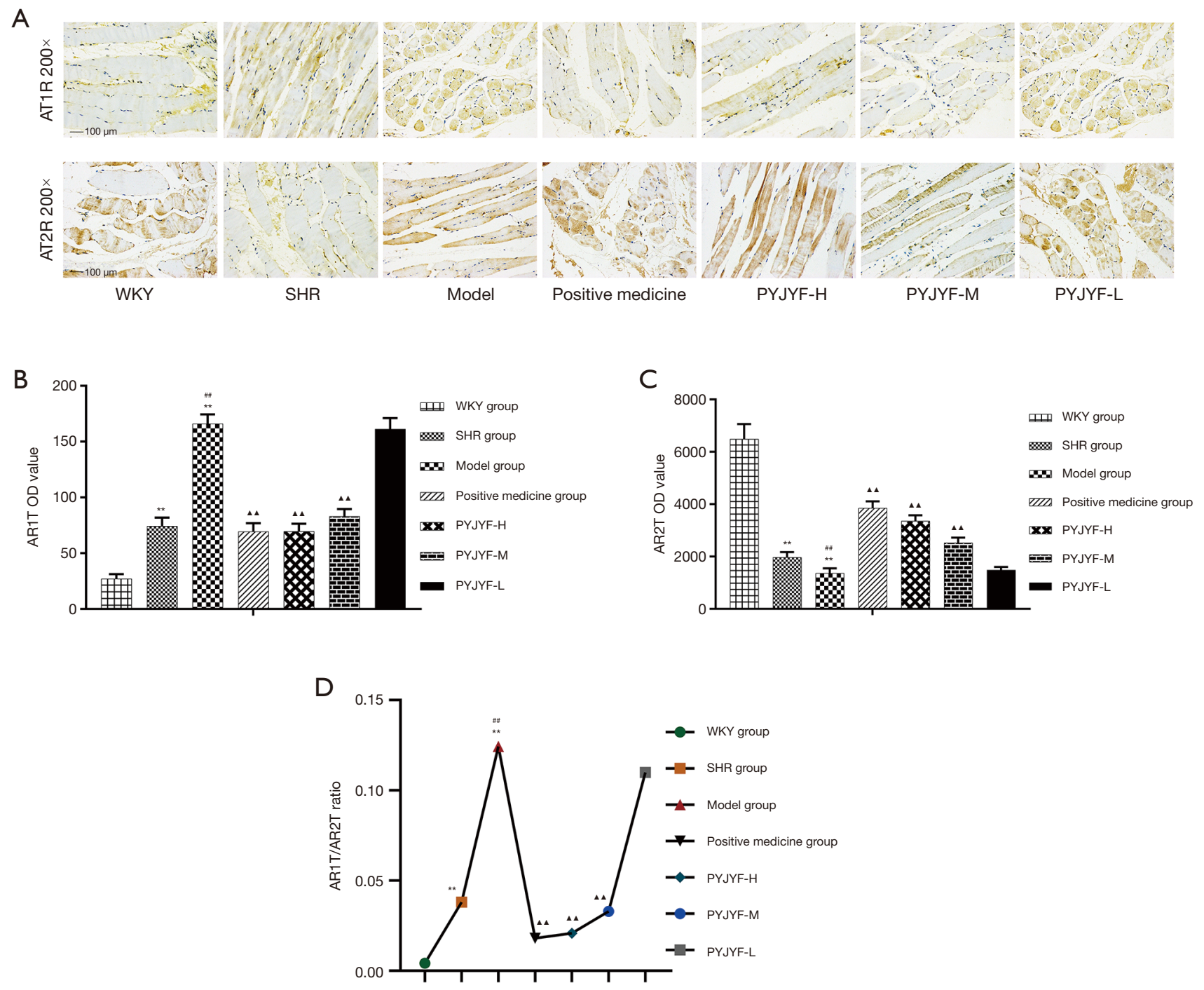

Figure 3 The effect of PYJYF on the expression of skeletal muscle AT1R and AT2R. (A) Representative graph of AT1R and AT2R immunohistochemical staining of rat skeletal muscle in each group; (B) AT1R OD value of rat skeletal muscle in each group; (C) AT2R OD value of rat skeletal muscle in each group; (D) average ratio of rat AT1R/AT2R ratio. All values are expressed as the means \pm SDs. Compared with WKY group, * $\mathrm{P}<0.05$, **, $\mathrm{P}<0.01$; compared with SHR group, ${ }^{*}, \mathrm{P}<0.05,{ }^{\# \#}, \mathrm{P}<0.01$; compared with the model group, , $\mathrm{P}<0.05,{ }^{\boldsymbol{\Delta}}$, $\mathrm{P}<0.01$. AT1R, angiotensin II type 1 receptor; AT2R, angiotensin II type 2 receptor; OD, optical density; WKY, Wistar-Kyoto rat (as a normal control); SHR, spontaneously hypertensive rats; PYJYF, Pingyang Jiangya Fang (a prescriptions of traditional Chinese medicine); PYJYF-H, PYJYF high dose group; PYJYF-M, PYJYF medium dose group; PYJYF-L, PYJYF low dose group.

group $(\mathrm{P}<0.01)$. Figure $3 D$ shows that the AT1R/ AT2R ratio was significantly lowered in the PYJYF-H, PYJYF-M, and positive medicine groups $(\mathrm{P}<0.01)$. This indicates that PYJYF significantly reduced AT1R and upregulated the expression of AT2R, reduced its affinity with its receptor Ang II, thereby lowering the blood pressure.

\section{PYYYF inbibits hyperactivity of RASS system}

The positive medicine group and PYJYF-H and PYJYF-M reduced the content of Pro-Ang II-Ald $(\mathrm{P}<0.01)$, inhibited the hyperactivity of the RASS system to dilate the small veins, reduced blood pressure and the amount of blood flow to the heart, as well as improved effective circulation (Figure 4A). 
A

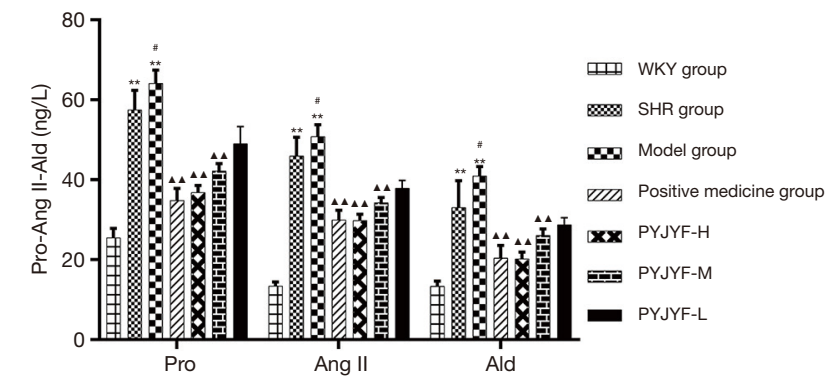

B

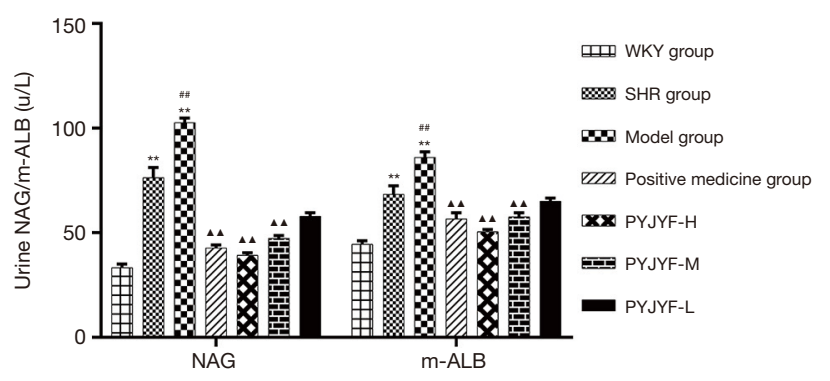

C
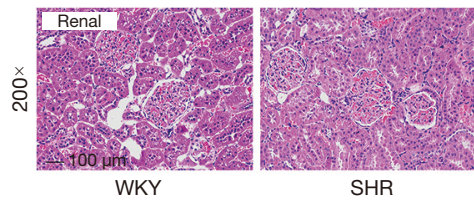

SHR
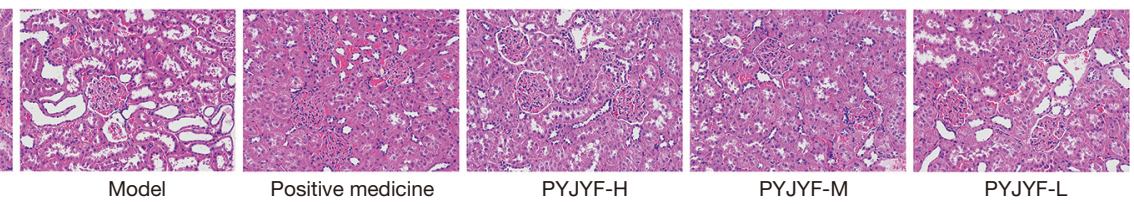

D
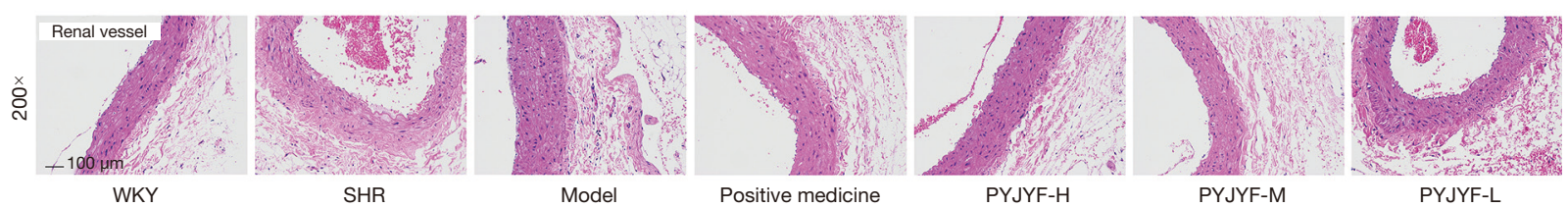

E
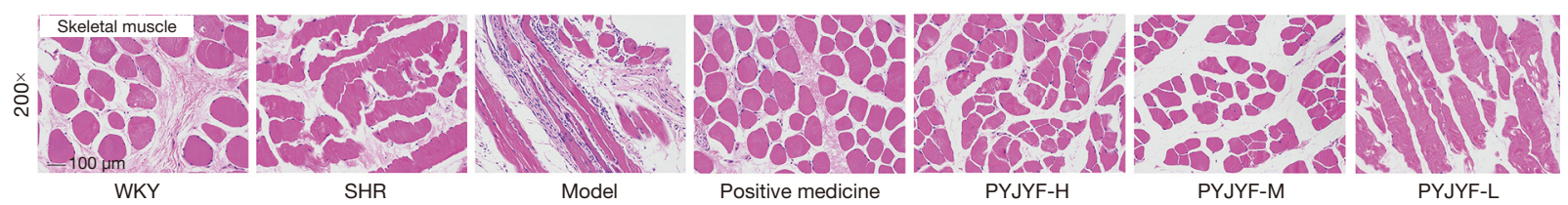

PYJYF-M
PYJYF-L

Figure 4 The effect of PYJYF on Pro-Ang II-Ald system, urine NAG and m-ALB, renal, renal blood vessels, skeletal muscle pathological tissues; (A) Pro, Ang II, Ald (ng/L) of rats in each group; (B) NAG and m-ALB of urine of rats in each group (u/L); (C) renal pathological tissue hematoxylin-eosin (H\&E) staining slices of rats in each group; (D) renal vascular pathological tissue hematoxylin-eosin (H\&E) staining slices of rats in each group; (E) skeletal pathological tissue hematoxylin-eosin (H\&E) staining slices of rats in each group. Compared with WKY group, *, $\mathrm{P}<0.05,{ }^{* *}, \mathrm{P}<0.01$; compared with SHR group, ${ }^{*}, \mathrm{P}<0.05,{ }^{\#}, \mathrm{P}<0.01$; compared with the model group, ${ }^{\boldsymbol{\Lambda}}, \mathrm{P}<0.05,{ }^{\mathbf{\Lambda}}, \mathrm{P}<0.01$. ProAng II-Ald, p-renin receptor-angiotensin-aldosterone; NAG, N-acetyl- $\beta$-D-glucosidase; m-ALB, microalbuminuria; WKY, Wistar-Kyoto rat (as a normal control); SHR, spontaneously hypertensive rats; PYJYF, Pingyang Jiangya Fang (a prescriptions of traditional Chinese medicine); PYJYF-H, PYJYF high dose group; PYJYF-M, PYJYF medium dose group; PYJYF-L, PYJYF low dose group.

\section{PYFYF reduced urine $N A G, m-A L B$}

Notably, urine NAG, m-ALB are sensitive indicators of renal damage of hypertension (15). Here, PYJYF-H, PYJYF-M, and the positive medicine group reduced urine NAG and $\mathrm{m}-\mathrm{ALB}$ content $(\mathrm{P}<0.01)$ (Figure $4 B)$.

\section{Protective effects of PYFYF on renal, renal vascular, skeletal muscle pathology}

Long-term hypertension causes renal damage. In addition to being the regulatory center of ROS, AT1R/AT2R expression, skeletal muscle is closely related to hypertension and IR (16). Renal pathological structure in the model group showed individual model glomerular atrophy, decreased cell number, compensatory hypertrophy of the surrounding glomeruli, and extensive expansion of the tubule lumen. PYJYF-H, PYJYF-M, and positive medicine group had clear cell boundaries, no significant increase in glomerular cells, and the renal tubular structure was normal (Figure 4C). 
The renal vascular pathological structure of the model group revealed mild hyperplasia of the intima of the blood vessels, hypertrophy of the media, and a small amount of new small blood vessels and plasma cell infiltration on the adventitia. PYJYF-H, PYJYF-M, and positive medicine group showed no apparent lesions on the vascular intima, the median structure was normal, the adventitia structure was normal, and there was no inflammation or angiogenesis (Figure 4D).

The pathological structure of the skeletal muscle in the model group showed that localized muscle fibers were reduced in muscle tissue, fibroblasts were proliferated, and tissue fibrosis was observed. Besides, a small amount of inflammatory cell infiltration was observed in the tissue. Whereas, PYJYF-H, PYJYF-M, and positive medicine group had clear cell boundaries of the muscle, the muscle fibers and fibroblasts did not decrease, proliferate, and fibrosis. The structure was normal (Figure 4E).

As such, this reflects the protective effects of PYJYF-H and PYJYF-M on renal, renal vessels, and skeletal muscle.

\section{PYFYF regulates the expression of ROS/Akt in skeletal muscle}

Ang II activates multiple intracellular signaling pathways by binding to AT1R and stimulates the generation of ROS in the ROS in the cell. Of note, ROS is a critical indicator for evaluating the vasoactive peptide Ang II (17). IR increased the oxidative stress response of the body and ROS production under high glucose conditions. Also, the Akt signaling pathway plays a vital role in high glucose-induced damage and is closely related to oxidase mediated generation. ROS is the junction of IR and skeletal muscle. When ROS was overproduced, the synthesis of collagen and fibrosis was promoted via the transcription factor protein kinase P13K/Akt. Herein, ROS content of the skeletal muscle was increased (Figure $5 A$ ), and the expression of Akt mRNA (Figure $5 B$ ), and protein (Figure $5 C$ ) were upregulated in the model group $(\mathrm{P}<0.05, \mathrm{P}<0.01)$. On the other hand, the expression of $\mathrm{P}-\mathrm{Akt} / \mathrm{Akt}$ protein was downregulated in the model group (Figure $5 C, \mathrm{P}<0.05, \mathrm{P}<0.01$ ). PYJYF-H, PYJYF-M, and positive medicine group significantly reduced the ROS content $(\mathrm{P}<0.01)$, downregulated the expression of Akt mRNA and protein $(\mathrm{P}<0.05, \mathrm{P}<0.01)$, as well as upregulated the expression of $\mathrm{P}$-Akt/Akt protein in skeletal muscle $(\mathrm{P}<0.05$, $\mathrm{P}<0.01)$.

\section{Discussion}

Hypertension is one of the most common diseases across the globe, and above $50 \%$ of patients diagnosed with hypertension have IR. Based on research reports, if a patient diagnosed with hypertension develops a disorder of glucose and lipid metabolism, there is a $95 \%$ incidence of IR (18). Furthermore, several studies have suggested IR as the common pathway of essential hypertension caused by genetics and the environment. As such, taking IR as a therapeutic target is of great significance for the occurrence, development, treatment, and prognosis of hypertension.

Historically, Chinese medicine identifies hypertension as "dizziness". The most prevalent clinical symptoms include dizziness, tinnitus, swollen head, redness eyes, irritability, heavy head and feet, red tongue, and strong pulse strings. Dry mouth can also be observed. Insufficient liver Yin, Yin does not control Yang, Liver Yang gas rising and floating hyperinversion is the basic pathological change of this syndrome. Traditional Chinese medicine clinically summarizes the above symptoms as Liver Yang hyperactivity syndrome, and uses Pinggan Qianyang as a treatment method. SHR rats and Otsuka longevans Tokushima faty (OLETF) rats are currently considered as ideal animal models of hypertension combined with IR. This is in line with the clinical characteristics of IR in hypertension (14). Interestingly, we found that the blood pressure of SHR rats significantly increased after the administration of Fuzi Decoction, besides, there was the presence of IR, as well as the behavioral of Liver-Yang hyperactivity syndrome. Chemically synthesized antihypertensive drugs cannot improve the Liver-Yang hyperactivity syndrome. Moreover, we found that PYJYF significantly improved the Liver-Yang hyperactivity syndrome and IR, however, its mechanism remains unclear. Thus, Liver-Yang hyperactivity syndrome will be among the objectives of our follow-up research.

In this study, PYJYF effectively improved the scleral redness, irritability, water intake, rotation time, and pain threshold in rats. Besides, its hypotensive effect was demonstrated in acute and chronic hypotensive experiments. FPG, FINs, RES, and HOMA-IR have been considered as markers of IR, and their changes explain the severity of hypertension with IR (19). At the same time, the contents of FPG, FINs, RES, and HOMA-IR significantly decreased after treatment with PYJYF. On the other hand, Pro, Ang II, Ald, urinary NAG, and urinary m-ALB were specific markers of hypertension RASS system and renal injury, respectively. In this work, PYJYF reduced the levels of Pro, 
A

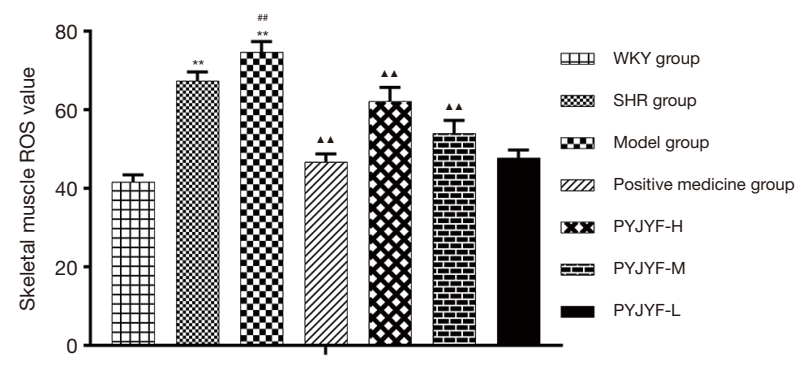

B

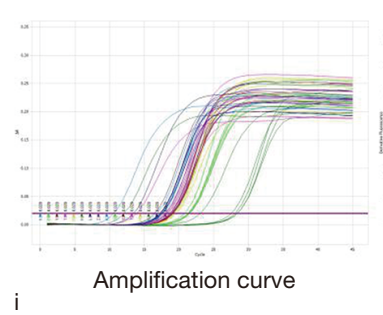

i
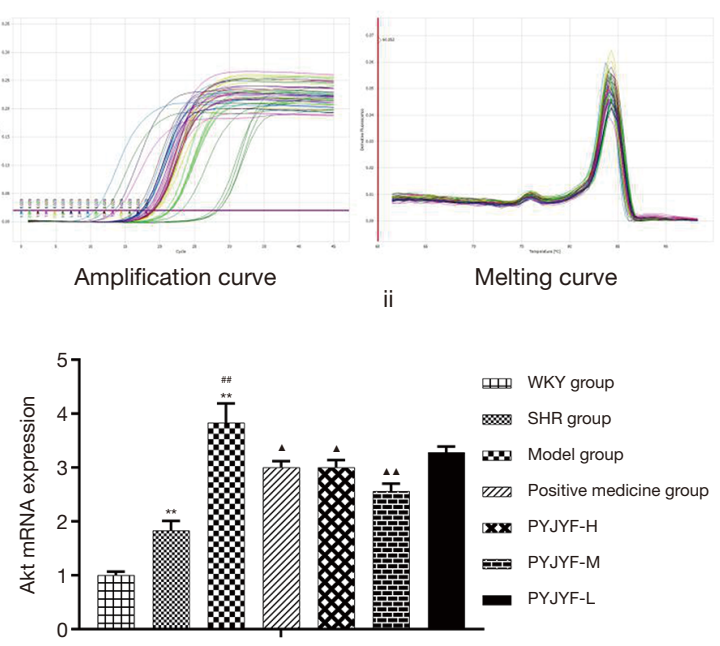

C
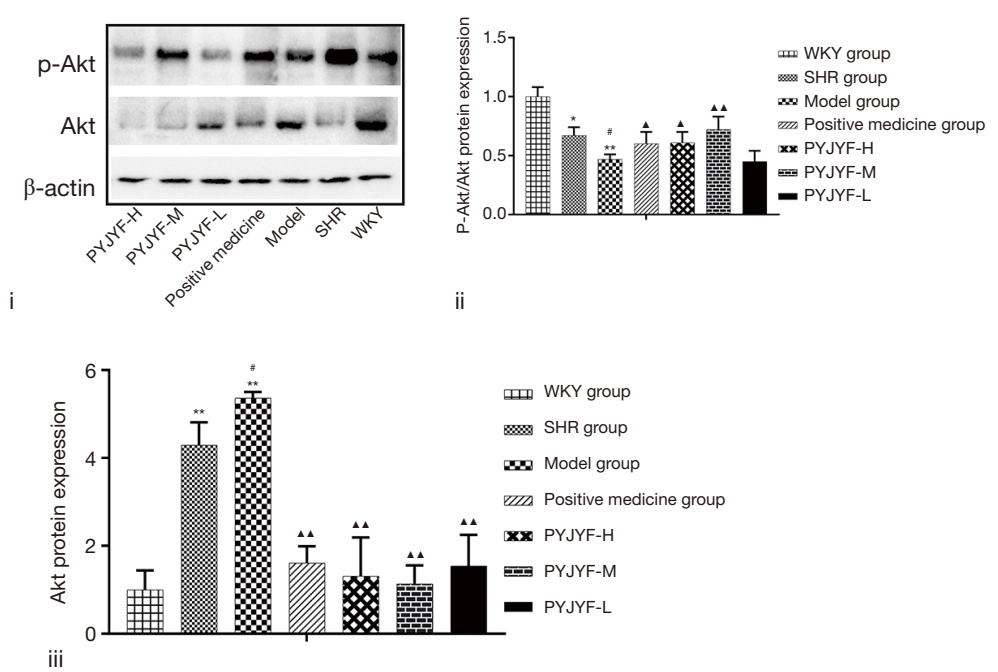

Figure 5 The effect of PYJYF on the expression of ROS/Akt signal; (A) ROS expression of skeletal muscle in each group; (B) Akt mRNA expression in each group of rats; (C) p-Akt/Akt protein expression in each group of rats. Compared with WKY group, *, $\mathrm{P}<0.05,{ }^{* *}, \mathrm{P}<0.01$; compared with SHR group, ${ }^{\prime \prime}, \mathrm{P}<0.05,{ }^{, \#}, \mathrm{P}<0.01$; compared with the model group, ${ }^{\mathbf{\Delta}}, \mathrm{P}<0.05,{ }^{\mathbf{\Lambda}}, \mathrm{P}<0.01$. ROS, reactive oxygen species; p-Akt, phosphorylated serine/threonine kinase; Akt, serine/threonine kinase; WKY, Wistar-Kyoto rat (as a normal control); SHR, spontaneously hypertensive rats; PYJYF, Pingyang Jiangya Fang (a prescriptions of traditional Chinese medicine); PYJYF-H, PYJYF high dose group; PYJYF-M, PYJYF medium dose group; PYJYF-L, PYJYF low dose group.

Ang II, Ald, urine NAG, and m-ALB, as well as inhibited hyperactivity of RASS thereby suggesting that renal damage was improved. The experimental results showed that PYJYF exhibits a satisfactory antihypertensive effect, improves IR, inhibits hyperactivity of the RASS system, and contributes to the prevention and treatment of renal damage.

Hypertensive patients suffer from varying degrees of glucose metabolism disorders due to a combination of poorly controlled blood pressure in hypertension, vascular endothelial damage, and IR (20). Nevertheless, they are clinically different, and their possible interaction mechanisms remain entangled or ignored. Persistent IR in hypertensive patients potentially trigger persistent oxidative stress (21), suggesting a potential oxidative stress mechanism for hypertension-related IR.
On one hand, insulin regulates blood sugar balance by promoting skeletal muscle and adipose tissue uptake of glucose as well as inhibiting liver glycogenogenesis. IR induces vascular endothelial cells to produce $\mathrm{NO}$, increase blood flow, and further increase glucose uptake by skeletal muscle. Notably, high glucose concentration causes skeletal muscle damage. On the other hand, nutrients are delivered to target organs by regulating microvascular nutrition, including cardio cerebral renal and neurovascular units $(22,23)$. Hypertension is a state of oxidative stress, resulting in the generation of excessive reactive oxygen. High blood pressure might cause long-term changes in the oxidative stress system, RASS system, IR, and nervous system, leading to the damage of the brain, renal, and neurovascular units in patients with hypertension. There exists several 
complications of diabetes, heart failure, renal failure, and stroke. However, the endocrine biological mechanism of this population is largely understudied. Therefore, taking IR as a therapeutic target and analyzing how to prevent and treat essential hypertension is a novel strategy for the treatment of hypertension. Furthermore, improving the Liver-Yang hyperactivity syndrome and normalizing insulin function is of great significance for the treatment of early hypertension and the reversal of various complications of hypertension.

Angiotensin II receptor blockers (ARBs) and their response to multiple challenges are discontinued in patients with hypertension. Because angiotensin II receptor blockers exhibit special advantages in improving IR and glucose metabolism disorders, they have been considered to be closely related to hypertension complications (24). Summarily, the RASS system and insulin function are repeatedly related to the development of hypertension and various complications. We found that the antihypertensive effect of PYJYF and angiotensin II receptor blockers were similar to the effect of IR, suggesting that PYJYF potentially acts by inhibiting the hyperactivity of the RASS system and reducing it. Also, it can be achieved by inhibiting the production of Ang II, improving IR, and reducing oxidative stress.

Hyperactivity of the RASS system causes damage to the renal and renal vessels. Notably, the renal and renal vessels are the key areas for regulating blood volume as well as maintaining the circulatory system and electrolyte balance. Ang II is the primary component of the RASS system. The antihypertensive effect of ARB protects the heart, brain, renal, and vascular structure, while the role of neurovascular units is vital in the prevention and treatment of hypertension.

Of note, AT1R and AT2R are in the RASS system and regulate blood pressure and hypertension complications. AT1R, being a primary receptor of Ang II, directly inhibits Ang II through ligand binding, reduces the effect of Ang II on insulin molecular signaling pathways, and improves Ang II-induced IR. On the other hand, AT2R increases the short-term and long-term effects of angiotensin on osmotic pressure and blood pressure, protects the heart, brain, renal and their neurovascular units, as well as prevents hypertension damage to the heart, brain, and renal $(25,26)$.

At the same time, AT1R and AT2R are the most abundant in skeletal muscle and negatively regulate the RASS system and insulin molecular signaling pathways (27). The hyperactivity of the RASS system triggers a continuous increase of Ang II, an upregulation in expression of AT1R, and a downregulation in expression of AT2R. The role of Ang II is primarily mediated by AT1R and AT2R. Under IR, the islet $\beta$ cell glucose metabolism function is reduced. High blood glucose cause an enhanced oxidative stress response of the body and ROS generation. Also, the Akt signaling pathway plays a vital role in high glucoseinduced damage and is closely related to oxidase mediated generation (28). Ang II stimulates multiple cell signals to the pathway while its downstream molecules stimulate cells to produce a large amount of ROS. Nonetheless, it only works on the Akt signaling pathway without influencing other signaling pathways under the effect of IR $(29,30)$. Ang II stimulates multiple cell signals to the pathway while its downstream molecules stimulate cells to produce a large amount of ROS. Nonetheless, it only works on the Akt signaling pathway without influencing other signaling pathways under the effect of IR (31). Additionally, Ang II activates NAPDH to increase intracellular ROS, induces Akt signaling pathways to regulate cell proliferation and growth, and promotes multiple physiological processes including glucose metabolism and apoptosis (32).

Under the action of Fuzi Decoction oxidative stress, SHR rats activated the ROS/Akt signaling pathway. The ROS/ Akt signaling pathway then acted on the skeletal muscle, the target tissue with the richest blood vessels, and the most active metabolism in the body. Excess insulin secretion promoted skeletal muscles to take up a large amount of glucose thereby disturbing the metabolism of glycolipid and protein, which influences blood supply and metabolism, as well as induces IR. IR excited the sympathetic nerve and L-arginine-NO pathway, increased blood flow of skeletal muscle tissue rich in small arteries, increased peripheral vascular resistance, and caused vasoconstriction and diastolic dysfunction. This therefore suggests a relationship between vascular resistance and IR. Moreover, excess insulin in the insulin-resistant body stimulated endothelial and smooth muscle cell endothelin-1 gene expression and a decrease in synthetic blood volume, activated the RASS, and upregulated the expression of peripheral Ang II. Further, it extensively expressed AT1R, AT2R in skeletal muscle as the primary receptor of Ang II, which is the target of skeletal muscle to increase vascular resistance. On the other hand, IR promoted sodium reabsorption by insulin in the distal renal tubules, reduced urine sodium excretion, venous reflux, and increased cardiac output. Additionally, it increased SBP under the action of vascular resistance.

In the present study, SHRs activated ROS/Akt signaling 
pathway under the action of Fuzi Decoction oxidative stress and induced IR. The rats developed clinical manifestations of Liver-Yang hyperactivity. Notably, PYJYF inhibited ROS/Akt signaling pathway, resisted IR, promoted insulin metabolism, corrected skeletal muscle glucose and fat as well as protein metabolism disorders. Also, it reduced peripheral blood volume, antagonized the RASS system, lowered the expression of peripheral Ang II, inhibited Ang II, and skeletal muscle cell AT1R, AT2R was bound by ligand to reduce vascular resistance. In addition, reduced insulin inhibited sodium reabsorption in the distal renal tubules, promoted urinary sodium excretion, reduced venous return and cardiac output thereby reducing SBP in SHRs. Studies also found that PYJYF exhibits a protective effect on the injury of target organs in the renal, renal vessels, and skeletal muscle tissues of SHR rats with LiverYang hyperactivity with the antihypertensive therapy mechanism of PYJYF on target organs being pathologically verified.

Although we explored the pathological mechanism of PYJYF through the ROS/Akt pathway to protect the target organ damage of essential hypertension. ROS/Akt pathway is one of the main molecular biological processes that inhibit cell apoptosis, but the mechanism of ROS/Akt pathway inhibiting cell apoptosis and protecting the blood vessels of essential hypertension is still unclear. ROS/Akt pathway protect vascular function and prevent cardiovascular at the occurrence of the event is of great significance, which is the focus of our next research.

\section{Conclusions}

In conclusion, our findings showed that the pathological structure of renal, renal vessels, and skeletal muscle appeared normal after PYJYF treatment. Moreover, the expression of AT1R, AT2R, and ROS/Akt were significantly downregulated. Therefore, these data indicate that PYJYF exerts a hypotensive effect by inhibiting oxidative stress.

At present, there exists no specific therapy for hypertension with Liver-Yang hyperactivity syndrome, and the combination of chemically synthesized antihypertensive drugs has shown different side effects. Notably, the traditional Chinese medicine prescription is characterized by a strong overall adjustment ability and unique treatment of complications. For instance, the antihypertensive and antihypertensive action of PYJYF originating from Tianma Gouteng prescription with a long history of health effects in China has been listed in the authoritative Chinese
Pharmacopoeia.

Our work reveals that PYJYFg improves the Liver-Yang hyperactivity syndrome to a certain extent, and exhibits a significant antihypertensive effect, hence protecting the structure and function of the renal, renal vessels, and skeletal muscle. Its underlying mechanism might be via inhibiting the ROS/Akt pathway oxidative stress response.

\section{Acknowledgments}

Funding: This study was financially supported by the National Natural Science Foundation of China (No. 81573965), National major new drug development project (2019ZX09301103), the Scientific research fund of Hunan administration of traditional Chinese medicine (201544), and the Provincial Department of Graduate research innovation project of Hunan (CX20190565).

\section{Footnote}

Reporting Checklist: The authors have completed the ARRIVE reporting checklist. Available at http://dx.doi. org/10.21037/apm-20-1371

Data Sharing Statement: Available at http://dx.doi. org/10.21037/apm-20-1371

Conflicts of Interest: All authors have completed the ICMJE uniform disclosure form (available at http://dx.doi. org/10.21037/apm-20-1371). The authors have no conflicts of interest to declare.

Ethical Statement: The authors are accountable for all aspects of the work in ensuring that issues related to the accuracy or integrity of any part of the work are appropriately investigated and resolved. All animal experiments were approved by the Animal Care and Ethical Committee of the First Affiliated Hospital of Hunan University of Traditional Chinese Medicine (Changsha, China), accepted number: ZYFY20160408. Procedures and protocols were performed in strict accordance with the Guide for the Care and Use of Laboratory Animals of the International Association for the Study of Pain (IASP).

Open Access Statement: This is an Open Access article distributed in accordance with the Creative Commons Attribution-NonCommercial-NoDerivs 4.0 International License (CC BY-NC-ND 4.0), which permits the non- 
commercial replication and distribution of the article with the strict proviso that no changes or edits are made and the original work is properly cited (including links to both the formal publication through the relevant DOI and the license). See: https://creativecommons.org/licenses/by-ncnd/4.0/.

\section{References}

1. Grundy SM, Stone NJ, Bailey AL, et al. 2018 AHA/ ACC/AACVPR/AAPA/ABC/ACPM/ADA/AGS/APhA/ ASPC/NLA/PCNA guideline on the management of blood cholesterol: a report of the American College of Cardiology/American Heart Association Task Force on Clinical Practice Guidelines. J Am Coll Cardiol 2019;73:e285-350.

2. Leimena C, Qiu H. Non-coding RNA in the pathogenesis, progression and treatment of hypertension. Int J Mol Sci 2018;19:927.

3. Dong H, Zhang S, Du W, et al. Pharmacodynamics and metabonomics study of Tianma Gouteng Decoction for treatment of spontaneously hypertensive rats with liver-yang hyperactivity syndrome. J Ethnopharmacol 2020;253:112661.

4. Tai J, Zou J, Zhang X, et al. Randomized controlled trials of Tianma Gouteng Decoction combined with nifedipine in the treatment of primary hypertension: a systematic review and meta-analysis. Evid Based Complement Alternat Med 2020;2020:5759083

5. Chen B, Wang Y, He Z, et al. Tianma Gouteng decoction for essential hypertension: Protocol for a systematic review and meta-analysis. Medicine (Baltimore) 2018;97:e9972.

6. Kochuieva M, Psarova V, Ruban L, et al. Associations of irs-1 polymorphism with various components of the metabolic syndrome in hypertensive patients. Wiad Lek 2019;72:1484-98.

7. Santana NM, Mendes RML, Silva NFD, et al. Sarcopenia and sarcopenic obesity as prognostic predictors in hospitalized elderly patients with acute myocardial infarction. Einstein (Sao Paulo) 2019;17:eAO4632.

8. Gao W, Wang W, Zhang J, et al. Allicin ameliorates obesity comorbid depressive-like behaviors: involvement of the oxidative stress, mitochondrial function, autophagy, insulin resistance and NOX/Nrf2 imbalance in mice. Metab Brain Dis 2019;34:1267-80.

9. Tata CM, Sewani-Rusike CR, Oyedeji OO, et al. Senecio serratuloides extract prevents the development of hypertension, oxidative stress and dyslipidemia in nitric oxide-deficient rats. J Complement Integr Med 2020. doi: 10.1515/jcim-2018-0073.

10. Vest AR, Young JB, Cho L. The metabolic syndrome, cardiovascular fitness and survival in patients with advanced systolic heart failure. Am J Cardiol 2018;122:1513-9.

11. Lee JH, Chan JL, Yiannakouris N, et al. Circulating resistin levels are not associated with obesity or insulin resistance in humans and are not regulated by fasting or leptin administration: cross-sectional and interventional studies in normal, insulin-resistant, and diabetic subjects. J Clin Endocrinol Metab 2003;88:4848-56.

12. Guo X, Fan G, Shi B, et al. Study on the relationship between resistin and its related factors and development of regression equation in type 2 diabetes mellitus. Lab Med 2005;20:455-8.

13. Surapongchai J, Rattanavichit Y, Buniam J, et al. Exercise protects against defective insulin signaling and insulin resistance of glucose transport in skeletal muscle of angiotensin II-infused rat. Front Physiol 2018;9:358.

14. Lu D, Peng F, Li J, et al. Urotensin II promotes secretion of LTB(4) through 5-lipoxygenase via the UT-ROSAkt pathway in RAW264.7 macrophages. Arch Med Sci 2019;15:1065-72.

15. Malhotra R, Katz R, Jotwani V, et al. Urine markers of kidney tubule cell injury and kidney function decline in SPRINT trial participants with CKD. Clin J Am Soc Nephrol 2020;15:349.

16. Paolillo FR, Mattos VS, Borghi-Silva A, et al. Advanced glycation endproducts as biomarkers for risk of diabetes and cardiovascular diseases by skin autofluorescence: a noninvasive optical screening. Photobiomodul Photomed Laser Surg 2019;37:168-74.

17. van Raalte DH, Bjornstad P. Role of sodium-glucose cotransporter 2 inhibition to mitigate diabetic kidney disease risk in type 1 diabetes. Nephrol Dial Transplant 2020;35:i24-32.

18. Wijaya IN, Athiyah U, Fasich, et al. Knowledge, attitude, and practice of pharmacists towards management of hypertension in primary care centers. J Basic Clin Physiol Pharmacol 2020. doi: 10.1515/jbcpp-2019-0319.

19. Rodríguez-Méndez AJ, Carmen-Sandoval W, LomasSoria C, et al. Timbe (Acaciella angustissima) pods extracts reduce the levels of glucose, insulin and improved physiological parameters, hypolipidemic effect, oxidative stress and renal damage in streptozotocin-induced diabetic rats. Molecules 2018;23:2812.

20. Yuan T, Yang T, Chen H, et al. New insights into oxidative stress and inflammation during diabetes mellitus- 
accelerated atherosclerosis. Redox Biol 2019;20:247-60.

21. Langer HT, Afzal S, Kempa S, et al. Nerve damage induced skeletal muscle atrophy is associated with increased accumulation of intramuscular glucose and polyol pathway intermediates. Sci Rep 2020;10:1908.

22. Brutman-Barazani T, Horovitz-Fried M, Aga-Mizrachi $\mathrm{S}$, et al. Protein kinase $\mathrm{C}$ but not PKC is involved in insulin-induced glucose metabolism in hepatocytes. J Cell Biochem 2012;113:2064-76.

23. Elkahloun AG, Saavedra JM. Candesartan neuroprotection in rat primary neurons negatively correlates with aging and senescence: a transcriptomic analysis. Mol Neurobiol 2020;57:1656-73.

24. Bea JW, Wassertheil-Smoller S, Wertheim BC, et al. Associations between ACE-inhibitors, angiotensin receptor blockers, and lean body mass in community dwelling older women. J Aging Res 2018;2018:8491092.

25. Philip AM, Wang Y, Mauro A, et al. Development of a zebrafish sepsis model for high-throughput drug discovery. Mol Med 2017;23:134-48.

26. Quiroga DT, Miquet JG, Gonzalez L, et al. Mice lacking angiotensin type 2 receptor exhibit a sex-specific attenuation of insulin sensitivity. Mol Cell Endocrinol

Cite this article as: $\mathrm{Li} \mathrm{Z,} \mathrm{Wu} \mathrm{M,} \mathrm{Liu} \mathrm{D,} \mathrm{Wang} \mathrm{X,} \mathrm{Yang} \mathrm{W,}$ Wang Y, Xiao C. Pingyang Jiangya Fang pretreatment reduces the blood pressure of spontaneously hypertensive rats with Liver-Yang hyperactivity syndrome via ROS/Akt oxidative stress pathway. Ann Palliat Med 2021;10(2):1904-1919. doi: 10.21037/ apm-20-1371
2019;498:110587.

27. Kohlhaas CF, Morrow VA, Jhakra N, et al. Insulin rapidly stimulates $\mathrm{L}$-arginine transport in human aortic endothelial cells via Akt. Biochem Biophys Res Commun 2011;412:747-51.

28. Sun B, Dong C, Lei H, et al. Propranolol inhibits proliferation and induces apoptosis of hemangioma-derived endothelial cells via Akt pathway by down-regulating Ang2 expression. Chem-Biol Interact 2020;316:108925.

29. Steinberg HO, Brechtel G, Johnson A, et al. Insulinmediated skeletal muscle vasodilation is nitric oxide dependent. A novel action of insulin to increase nitric oxide release. J Clin Invest 1994;94:1172-9.

30. Akhtar A, Sah SP. Insulin signaling pathway and related molecules: Role in neurodegeneration and Alzheimer's disease. Neurochem Int 2020;135:104707.

31. Frantz EDC, Prodel E, Braz ID, et al. Modulation of the renin-angiotensin system in white adipose tissue and skeletal muscle: focus on exercise training. Clin Sci (Lond) 2018;132:1487-507.

32. Luc K, Schramm-Luc A, Guzik T, et al. Oxidative stress and inflammatory markers in prediabetes and diabetes. $\mathrm{J}$ Physiol Pharmacol 2019;70:809-24. 